See discussions, stats, and author profiles for this publication at: https://www.researchgate.net/publication/326645407

\title{
On the MIMO Capacity with Multiple Linear Transmit Covariance Constraints
}

Conference Paper · June 2018

DOI: $10.1109 /$ NTCSpring.2018.8417596

CITATION

5 authors, including:

Ronan Farrell

National University of Ireland, Maynooth

188 PUBLICATIONS 603 CITATIONS

SEE PROFILE

(2) Le-Nam Tran

1 University College Dublin

100 PUBLICATIONS 1,683 CITATIONS

SEE PROFILE

Some of the authors of this publication are also working on these related projects:

Project $\quad$ Cell-Free Massive MIMO View project

Project Flexible Modulation Techniques for use in $5 \mathrm{G}$ communication systems View project
READS

124

Mark F. Flanagan

University College Dublin

136 PUBLICATIONS 655 CITATIONS

SEE PROFILE 


\title{
On the MIMO Capacity with Multiple Linear Transmit Covariance Constraints
}

\author{
Thuy M. Pham*, Ronan Farrell*, Holger Claussen ${ }^{\dagger}$, Mark Flanagan ${ }^{\ddagger}$, and Le-Nam Tran ${ }^{\ddagger}$ \\ *Department of Electronic Engineering, Maynooth University, Ireland. Email: \{minhthuy.pham, ronan.farrell $\} @$ mu.ie \\ ${ }^{\dagger}$ Nokia Bell Labs, Ireland. Email: holger.claussen@nokia-bell-labs.com. \\ ${ }^{\ddagger}$ School of Electrical and Electronic Engineering, University College Dublin, Ireland. Email: \{mark.flanagan,nam.tran\}@ucd.ie
}

\begin{abstract}
This paper presents an efficient approach to computing the capacity of multiple-input multiple-output (MIMO) channels under multiple linear transmit covariance constraints (LTCCs). LTCCs are general enough to include several special types of power constraints as special cases such as the sum power constraint (SPC), per-antenna power constraint (PAPC), or a combination thereof. Despite its importance and generality, most of the existing literature considers either SPC or PAPC independently. Efficient solutions to the computation of the MIMO capacity with a combination of SPC and PAPC have been recently reported, but were only dedicated to multipleinput single-output (MISO) systems. For the general case of LTCCs, we propose a low-complexity semi-closed-form approach to the computation of the MIMO capacity. Specifically, a modified minimax duality is first invoked to transform the considered problem in the broadcast channel into an equivalent minimax problem in the dual multiple access channel. Then alternating optimization and concave-convex procedure are utilized to derive water-filling-based algorithms to find a saddle point of the minimax problem. This is different from the state-of-the-art solutions to the considered problem, which are based on interiorpoint or subgradient methods. Analytical and numerical results are provided to demonstrate the effectiveness of the proposed low-complexity solution under various MIMO scenarios.

Index Terms-MIMO, linear transmit covariance constraints, sum power constraint, per-antenna power constraint, minimax duality, concave-convex procedure.
\end{abstract}

\section{INTRODUCTION}

The capacity of a Gaussian multiple-input multiple-output (MIMO) channel under a sum power constraint (SPC) or perantenna power constraint (PAPC) has been studied extensively [1]-[8]. While the former is apparently due to power budget or regulations, the latter is considered to avoid nonlinear distortions of the power amplifier associated with each transmit antenna. In practice, other power constraints can also be imposed on a MIMO system, not necessarily limited to SPC or PAPC separately. For example, optimal transmit covariance for multiple-input single output (MISO) channels with joint SPC and PAPC was studied in [9], [10]. In the context of cognitive networks, interference temperature constraints can be imposed on a secondary user (SU) to limit the interference generated at a primary user (PU) [11]-[13]. All of these constraints can be generally modeled as linear transmit covariance constraints (LTCCs) [11].

Efficient solutions to the computation of MIMO capacity with either SPC or PAPC have been well-studied [1], [2],
[5], [6], [8]. More recent research efforts have been made to characterize the capacity of Gaussian MIMO channels with joint SPC and PAPC [9], [10]. However, the work of [9] is only applicable to MISO systems, while that of [10] partially addresses general MIMO channels. For the general form of LTCCs, interior-point and subgradient methods were presented in [11], [12] to compute optimal transmit covariance matrices. However, it was demonstrated in [14] that these high-complexity methods are not useful for massive MIMO systems.

In this paper we propose efficient methods for computing the capacity of a single user MIMO (SU-MIMO) system under multiple LTCCs in the most general form. The channel state information is assumed to be known at both the transmitter and the receiver. In particular, we first transform the considered problem in the broadcast channel (BC) into an equivalent minimax problem in the dual multiple access channel (MAC), generalizing several results on the BC-MAC duality in the previous studies of [7], [11], [15]. In fact, a mimimax optimization approach was also considered in [7] but by interior-point algorithms. To find a saddle point of the considered minimax problem, we combine alternating optimization ( $\mathrm{AO})$ and concave-convex procedure (CCP) to arrive at an iterative algorithm, where each iteration is based on closed-form expressions. Our contributions are summarized as follows:

- We generalize the BC-MAC duality for an arbitrary number of LTCCs. In this way, the capacity of the BC with multiple LTCCs can be equivalently expressed as a minimax optimization problem in the dual MAC. The objective of the minimax problem is a concave-convex function of transmit and noise covariance matrices, respectively.

- We then propose a closed-form approach to computing a saddle point of the minimax problem by efficiently combining AO and CCP. The idea is to alternately optimize the transmit and noise covariance matrices, which is indeed a standard routine drawn on AO. For minimax problems, the convergence of $\mathrm{AO}$ is not guaranteed in general. The novelty of our proposed method is to optimize a bound of the objective obtained from the CCP when optimizing the noise covariance matrix. The 
proposed algorithm is provably convergent.

- We provide numerical results on the capacity of largescale MIMO systems with multiple LTCCs, which have not been reported previously.

Notation: Standard notations are used in this paper. Bold lower and upper case letters represent vectors and matrices, respectively. $\mathbf{I}_{N}$ defines an identity matrix of size $N ; \mathbf{I}$ and $\mathbf{0}$ define identity and zero matrices respectively, of which the size can be easily inferred from the context. $\mathbb{C}^{M \times N}$ denotes the space of $M \times N$ complex matrices; $\mathbf{H}^{\dagger}$ and $\mathbf{H}^{T}$ are Hermitian and ordinary transpose of $\mathbf{H}$, respectively; $\mathbf{H}_{i, j}$ is the $(i, j)$ entry of $\mathbf{H} ;|\mathbf{H}|$ is the determinant of $\mathbf{H}$; $\operatorname{diag}(\mathbf{x})$ denotes the diagonal matrix having diagonal entries matching the vector $\mathbf{x}$; $\operatorname{diag}(\mathbf{H})$, where $\mathbf{H}$ is a square matrix, is the vector of diagonal elements of $\mathbf{H} ; \operatorname{tr}(\mathbf{H})$ is the trace of a square matrix $\mathbf{H}$. Furthermore, we denote the expected value of a random variable by $\mathbb{E}[$.$] , and [x]_{+}=\max (x, 0)$. The $i$ th unit vector (i.e., its $i$ th entry is equal to one and all other entries are zero) is denoted by $\mathbf{e}_{i}$.

\section{System MODEL}

We consider a SU-MIMO model, where the transmitter and the receiver are equipped with $N$ and $M$ antennas, respectively. Note that a transmitter can obtain channel knowledge through receiver feedback or reciprocity of the MAC and BC. Therefore, we assume in this paper that the channel state information is perfectly known at the transmitter and the receiver. The received signal is given by

$$
\mathbf{y}=\mathbf{H s}+\mathbf{z}
$$

where $\mathbf{s}$ is the vector of transmitted symbols, and $\mathbf{z} \in \mathbb{C}^{M \times 1}$ is the background noise with distribution $\mathcal{C N}\left(\mathbf{0}, \mathbf{I}_{M}\right)$. Let $\mathbf{S}=\mathbb{E}\left[\mathbf{S s}^{\dagger}\right]$ be the input covariance matrix for the transmitted signal. We are interested in finding the capacity of the above channel with multiple LTCCs, which is formulated as

$$
\begin{array}{cl}
\underset{\mathbf{S} \succeq \mathbf{0}}{\operatorname{maximize}} & \log \left|\mathbf{I}+\mathbf{H S H}^{\dagger}\right| \\
\text { subject to } & \operatorname{tr}\left(\mathbf{E}_{i} \mathbf{S}\right) \leq P_{i}, i=1,2, \ldots, L
\end{array}
$$

where $\mathbf{E}_{i} \succeq \mathbf{0}$ is a predefined positive semidefinite matrix and $P_{i}$ is the corresponding power constraint, $L$ is the number of power constraints. Note that $(2 b)$ is called general linear constraints on the transmit covariance and it can include several types of transmit power constraints as special cases. Some examples are given below:

- If $\mathbf{E}_{i}$ is an identity matrix, the resulting constraint (2b) becomes a SPC.

- If $\mathbf{E}_{i}=\operatorname{diag}\left(\mathbf{e}_{i}\right)$, where $i=1,2, \ldots, N$ and $\mathbf{e}_{i}$ is a vector of all zeros except a value of one at the $i$ th position, the constraint reduces to a PAPC.

- If $\mathbf{E}_{i}=\mathbf{G G}^{\dagger}$, where $\mathbf{G}$ is the effective channel between a SU and the PU, then the resulting constraint limits the overall interference experienced by the PU.

In this paper we assume that $\mathbf{E}_{i}$ 's are introduced in such a way that the feasible set of (2) is bounded, and thus (2) is solvable. Since (2) is in fact a convex problem, it can be solved by a proper general-purpose optimization software. However, the computational complexity of convex solvers, which are mostly based on interior-point methods, increases dramatically with the problem size, and thus is not suitable for largescale MIMO systems. Hence, we propose a low-complexity iterative algorithm which will be numerically shown to achieve a superlinear convergence rate.

\section{Proposed Algorithm}

\section{A. Algorithm Description}

The focus of our paper is to transform the convex problem in the $\mathrm{BC}$ into an equivalent minimax problem in the MAC for which an efficient algorithm is derived. First we assume that the Slater condition holds for (2) which can be achieved if $P_{i}>0$ for all $i$. As a result, the duality gap is zero and (2) can be optimally solved in the dual domain. In this regard, let us denote $\mathbf{q}=\left[q_{1}, q_{2}, \ldots, q_{L}\right]^{T}$ be the vector of the Lagrange multipliers of (2) and $\mathbf{p}=\left[P_{1}, P_{2}, \ldots, P_{L}\right]^{T}$ be the corresponding power constraints. The proposed algorithm is based on the following theorem.

Theorem 1. The Lagrangian dual problem of (2) is equivalent to the following minimax problem

$$
\begin{aligned}
\min _{\mathbf{q} \geq 0} \max _{\overline{\mathbf{S}} \succeq \mathbf{0}} & \log \frac{\left|\sum_{i=1}^{L} q_{i} \mathbf{E}_{i}+\mathbf{H}^{\dagger} \overline{\mathbf{S}} \mathbf{H}\right|}{\left|\sum_{i=1}^{L} q_{i} \mathbf{E}_{i}\right|} \triangleq f(\mathbf{q}, \overline{\mathbf{S}}) \\
\text { subject to } & \operatorname{tr}(\overline{\mathbf{S}}) \leq P ; \mathbf{p}^{T} \mathbf{q} \leq P
\end{aligned}
$$

where $P=\sum_{i=1}^{L} P_{i}, \overline{\mathbf{S}}$ is the covariance matrix in the MAC.

Proof: See the Appendix.

We remark that the above result is in fact a generalization of several results for LTCCs presented in previous studies [7], [11], [15]. Without loss of optimality, the inequalities of (3) can be replaced with equalities. Let us define $\mathcal{Q} \triangleq$ $\left\{\mathbf{q} \mid \mathbf{q} \geq \mathbf{0}, \mathbf{p}^{T} \mathbf{q}=P\right\}$ and $\mathcal{S}=\{\overline{\mathbf{S}} \mid \overline{\mathbf{S}} \succeq \mathbf{0}, \operatorname{tr}(\overline{\mathbf{S}})=P\}$, we note that $f(\mathbf{q}, \overline{\mathbf{S}})$ is concave with $\overline{\mathbf{S}}$, and convex with $\mathbf{q}$, and twice differentiable. Thus a saddle point $\left(\mathbf{q}^{*}, \overline{\mathbf{S}}^{*}\right)$ exists for the minimax problem of (3) and it holds that

$$
f\left(\mathbf{q}^{*}, \overline{\mathbf{S}}\right) \leq f\left(\mathbf{q}^{*}, \overline{\mathbf{S}}^{*}\right) \leq f\left(\mathbf{q}, \overline{\mathbf{S}}^{*}\right) .
$$

For this general minimax problem, pure AO is not guaranteed to converge [7]. On the other hand, interior-point methods are possible but their complexity increases rapidly with the problem size, thus they are not attractive for large-scale MIMO systems [14].

In this paper we propose an iterative method that combines $\mathrm{AO}$ and CCP to solve (3) efficiently. The proposed method can be summarized as follows:

- For a given $\mathbf{q}$, we maximize $f(\mathbf{q}, \overline{\mathbf{S}})$ with respect to $\overline{\mathbf{S}}$, which can be solved efficiently by the classical waterfilling algorithm.

- For a given $\overline{\mathbf{S}}$, we minimize a convex upper bound of $f(\mathbf{q}, \overline{\mathbf{S}})$ which is obtained from the CCP. The novelty of the proposed method lies in the use of a convex upper bound, which is proved to generate a decreasing sequence of objective values. This method avoids fluctuations in a pure AO algorithm, and thus convergence is guaranteed. 
In the following we provide the details of the above steps. Let $\mathbf{q}^{n}$ be the value of $\mathbf{q}$ at the $n$th iteration of the proposed method and $\mathbf{Q}_{n}=\sum_{i=1}^{L} q_{i}^{n} \mathbf{E}_{i}$. Then, $\overline{\mathbf{S}}_{n}$ is the solution to the following problem

$$
\begin{array}{cl}
\underset{\overline{\mathbf{S}} \succeq \mathbf{0}}{\operatorname{maximize}} & \log \left|\mathbf{Q}_{n}+\mathbf{H}^{\dagger} \overline{\mathbf{S}} \mathbf{H}\right| \\
\text { subject to } & \operatorname{tr}(\overline{\mathbf{S}})=P .
\end{array}
$$

The problem above admits the water-filling solution [1], [2]. More explicitly, $\overline{\mathbf{S}}_{n}$ can be found as

$$
\overline{\mathbf{S}}_{n}=\mathbf{U}_{n} \hat{\mathbf{\Sigma}}_{n} \mathbf{U}_{n}^{\dagger}
$$

where $\mathbf{U}_{n} \boldsymbol{\Sigma}_{n} \mathbf{U}_{n}^{\dagger}=\mathbf{H Q}_{n}^{-1} \mathbf{H}^{\dagger}, \hat{\boldsymbol{\Sigma}}_{n}=\left[\mu \mathbf{I}-\boldsymbol{\Sigma}_{n}^{-1}\right]_{+}$and $\mu$ is the water-level, which satisfies the total power constraint

$$
\sum_{i=1}^{r}\left[\mathbf{H Q}_{n}^{-1} \mathbf{H}^{\dagger}\right]+=P
$$

where $r$ is the rank of the matrix $\mathbf{H Q}_{n}^{-1} \mathbf{H}^{\dagger}$. As mentioned earlier, monotonic convergence cannot be guaranteed by pure AO, which simply alternates between maximization and minimization of the same objective function. Herein, to avoid fluctuations, we propose using an upper bound of the objective for the minimization. In light of the CCP, we note that $f(\mathbf{q}, \overline{\mathbf{S}})$ in (3) can be expressed as a difference of two convex functions. Particularly, by the concavity property of the logdet function, we have

$$
\log \left|\mathbf{Q}+\mathbf{H}^{\dagger} \overline{\mathbf{S}}_{n} \mathbf{H}\right| \leq \log \left|\boldsymbol{\Phi}_{n}\right|+\operatorname{tr}\left(\boldsymbol{\Phi}_{n}^{-1}\left(\mathbf{Q}-\mathbf{Q}_{n}\right)\right)
$$

which produces

$$
f\left(\mathbf{q}, \overline{\mathbf{S}}_{n}\right) \leq \log \left|\boldsymbol{\Phi}_{n}\right|+\operatorname{tr}\left(\boldsymbol{\Phi}_{n}^{-1}\left(\mathbf{Q}-\mathbf{Q}_{n}\right)\right)-\log |\mathbf{Q}|
$$

where $\boldsymbol{\Phi}_{n}=\mathbf{Q}_{n}+\mathbf{H}^{\dagger} \overline{\mathbf{S}}_{n} \mathbf{H}$ and $\mathbf{Q} \triangleq \sum_{i=1}^{L} q_{i} \mathbf{E}_{i}$. The right hand side of (9) is a convex upper bound of the objective. To find $\mathbf{q}^{n+1}$ we solve the minimization of the upper bound. Since $\log \left|\boldsymbol{\Phi}_{n}\right|$ is a constant in this regard, $\mathbf{q}^{n+1}$ is in fact the solution to the following problem

$$
\begin{array}{cl}
\underset{\mathbf{q} \geq \mathbf{0}}{\operatorname{minimize}} & \operatorname{tr}\left(\boldsymbol{\Phi}_{n}^{-1} \mathbf{Q}\right)-\log |\mathbf{Q}| \\
\text { subject to } & \mathbf{p}^{T} \mathbf{q}=P
\end{array}
$$

or equivalently,

$$
\begin{aligned}
\underset{\mathbf{q} \geq 0}{\operatorname{minimize}} & \sum_{i=1}^{L} q_{i} \phi_{n, i}-\log \left|\sum_{i=1}^{L} q_{i} \mathbf{E}_{i}\right| \triangleq g(\mathbf{q}) \\
\text { subject to } & \mathbf{p}^{T} \mathbf{q}=P
\end{aligned}
$$

where $\phi_{n, i}=\operatorname{tr}\left(\boldsymbol{\Phi}_{n}^{-1} \mathbf{E}_{i}\right)$.

Remark 1. Our idea of using a convex upper bound for minimizing a cost function has a deep root in the successive convex approximation (SCA) framework. However, in the context of $\mathrm{SCA}$, the objective to be minimized is often nonconvex. In the considered problem, $f(\mathbf{q}, \overline{\mathbf{S}})$ is indeed convex with respect to $\mathbf{q}$ but an upper bound can be derived easily following the CCP. We note that other upper bounds can also be used in the proposed algorithm, as long as they meet the other conditions as well (see Property A of [16] for the detail). The upper bound found in (9) is relatively straightforward but it results in efficient methods for solving (11) as shown next.

In the general case of LTCCs, the gradient projection or conjugate gradient projection method can be utilized to solve (11) efficiently. The reason is that the feasible set of (11) is a simplex, and projection onto a simplex admits water-fillinglike algorithms [17]. A gradient projection based algorithm for solving (11) is described in Algorithm 1. When only PAPC is considered, a closed-form method for solving (11) was proposed in [8]. For the special case of joint SPC and PAPC, a closed-form solution for (11) is provided in the next subsection.

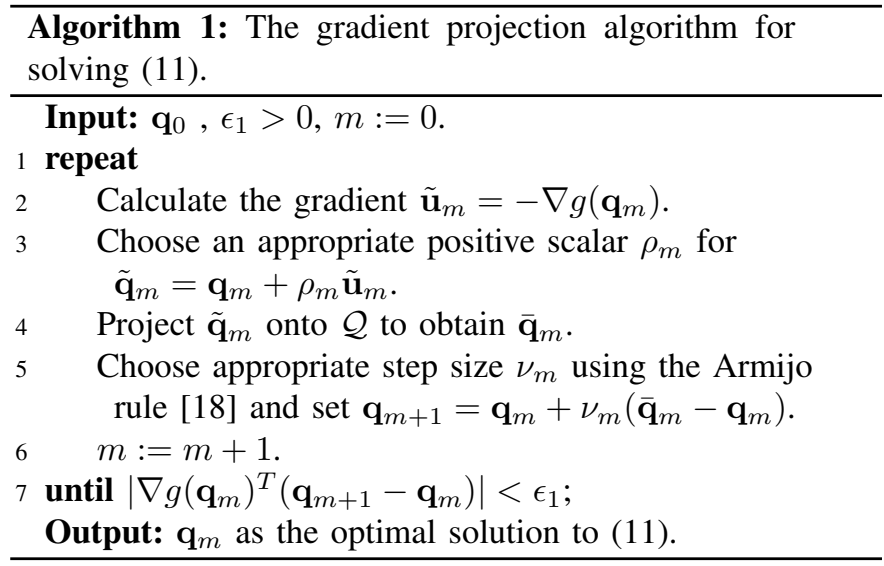

The complete algorithm to find the optimal transmit covariance matrix with multiple LTCCs is summarized in Algorithm 2. The convergence proof of Algorithm 2 follows the same arguments as those in [8], and thus is omitted here due to space limitations. The interested reader is referred to [8] for more details.

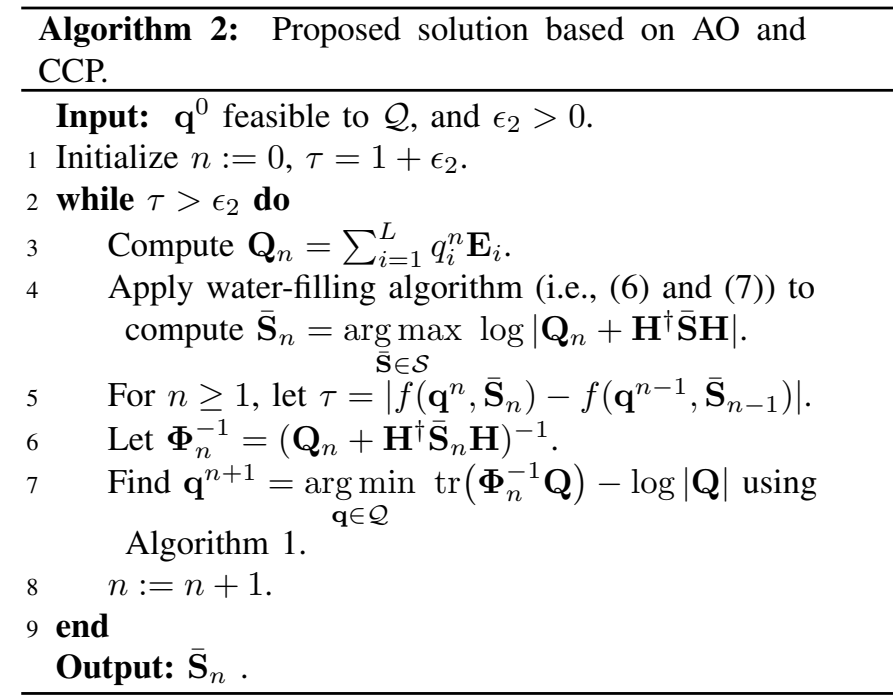

\section{B. MIMO Capacity with Joint SPC and PAPC}

For the special case of joint SPC and PAPC that has received growing interest recently [9], [10], we provide another method 
to solve (11) by closed-form expressions. However, no efficient method was proposed for this special case for the general MIMO setups in these two papers. In this case $L=N+1$ and we assume $\mathbf{E}_{N+1}=\mathbf{I}_{N}$ which represents the SPC, and $\mathbf{E}_{i}=\operatorname{diag}\left(\mathbf{e}_{i}\right)$ for $i=1,2, \ldots, N$ which represents the PAPC. In this way $P_{N+1}$ is the total transmit power $P_{T}$, and $P_{i}$, $i=1,2, \ldots, N$ is the power constraint for the $i$ th antenna.

We only consider the non-trivial case where $\min \left\{P_{i}\right\}<$ $P_{N+1}<\sum_{i=1}^{N} P_{i}$. If $P_{N+1} \leq \min _{1<i<N}\left\{P_{i}\right\}$, it is easy to see that (2) reduces to the MIMO capacity with a single SPC. Similarly, if $P_{N+1} \geq \sum_{i=1}^{N} P_{i}$, the SPC can be omitted and thus (2) becomes the MIMO capacity with PAPC [8].

It is trivial to see that in this case, problem (11) may be equivalently rewritten as

$\underset{\mathbf{q} \geq 0}{\operatorname{minimize}} q_{N+1} \phi_{n, N+1}+\sum_{i=1}^{N}\left(q_{i} \phi_{n, i}-\log \left(q_{N+1}+q_{i}\right)\right)$ subject to $\sum_{i=1}^{N+1} P_{i} q_{i}=P$.

The Lagrangian function of (12) is given by

$$
\begin{aligned}
& L(\mathbf{q}, \gamma)=q_{N+1} \phi_{n, N+1}+\sum_{i=1}^{N} q_{i} \phi_{n, i} \\
& \quad-\sum_{i=1}^{N} \log \left(q_{N+1}+q_{i}\right)+\gamma\left(\sum_{i=1}^{N+1} q_{i} P_{i}-P\right)
\end{aligned}
$$

where $\gamma$ is the multiplier. The dual function of (12) is

$$
g(\gamma)=\min _{\mathbf{q} \geq 0} L(\mathbf{q}, \gamma)
$$

For a given $\gamma$, note that the maximization of $L(\mathbf{q}, \gamma)$ over $q_{i}$, $i=1, \ldots N$, can be done in parallel, motivating us to apply the nonlinear Gauss-Seidel method [19] to compute $g(\gamma)$. In what follows, we use the subscript $m$ to denote the Gauss-Seidel iteration index. In particular, given the current iterate $\mathbf{q}_{m}=$ $\left[q_{m, 1}, q_{m, 2}, \ldots, q_{m, N+1}\right]^{T}$, we have the following iteration

$$
\begin{gathered}
q_{m+1, i}=\left[\frac{1}{\phi_{n, i}+\gamma P_{i}}-q_{m, N+1}\right]_{+}, i=1, \ldots, N \\
q_{m+1, N+1}=\left[x^{*}\right]_{+}
\end{gathered}
$$

where $x^{*}$ is the root of the equation

$$
\phi_{n, N+1}+\gamma P_{N+1}-\sum_{i=1}^{N} \frac{1}{x+q_{m+1, i}}=0 .
$$

The above equation can be solved easily by the Newton method or bisection method [19].

We also notice that $\boldsymbol{\Phi}_{n}^{-1}$ and the eigenvalue decomposition (EVD) of $\mathbf{H Q}_{n}^{-1} \mathbf{H}^{\dagger}$ can be computed with reduced complexity. Specifically let $\mathbf{G R}=\mathbf{H}$ be the $\mathrm{QR}$ decomposition of $\mathbf{H}$. If the singular value decomposition (SVD) of the upper triangular matrix $\mathbf{R} \mathbf{Q}_{n}^{-1 / 2}$ is $\tilde{\mathbf{U}}_{n} \tilde{\boldsymbol{\Sigma}}_{n} \tilde{\mathbf{V}}_{n}^{\dagger}=\mathbf{R} \mathbf{Q}_{n}^{-1 / 2}$, the EVD of $\mathbf{H Q}_{n}^{-1} \mathbf{H}^{\dagger}$ is simply given by $\mathbf{U}_{n}^{n} \boldsymbol{\Sigma}_{n} \mathbf{U}_{n}^{\dagger}=\mathbf{H Q}_{n}^{-1} \mathbf{H}^{\dagger}$, where $\mathbf{U}_{n}=\mathbf{G} \tilde{\mathbf{U}}_{n}$ and $\boldsymbol{\Sigma}_{n}=\tilde{\boldsymbol{\Sigma}}_{n}^{2}$. In addition, we can apply the matrix-inversion lemma [20] to compute $\boldsymbol{\Phi}_{n}^{-1}$. Let $\dot{\boldsymbol{\Sigma}}_{n}$ be the diagonal matrix containing all strictly positive entries of $\hat{\boldsymbol{\Sigma}}_{n}$, and $\dot{\mathbf{V}}_{n}$ be the corresponding singular vectors. After some manipulations, we arrive at $\boldsymbol{\Phi}_{n}^{-1}=\mathbf{Q}_{n}^{-1}-\mathbf{Q}_{n}^{-1 / 2} \dot{\mathbf{V}}_{n}\left(\dot{\boldsymbol{\Sigma}}_{n}^{-1}+\right.$
$\mathbf{I})^{-1} \dot{\mathbf{V}}_{n}^{\dagger} \mathbf{Q}_{n}^{-1 / 2}$ in which the inversion of the diagonal matrices $\dot{\boldsymbol{\Sigma}}_{n}^{-1}+\mathbf{I}$ and $\mathbf{Q}_{n}$ can be computed easily. Following similar arguments to those in [8], we can obtain a per-iteration complexity of $6\left(2 M N^{2}+11 N^{3}\right)$ and $6\left(2 N M^{2}+11 M^{3}\right)$ for the cases $M \geq N$ and $M<N$, respectively.

\section{The Special Case of MISO Capacity with Joint SPC and PAPC}

As mentioned earlier, this special case was recently studied in [9], [10]. We now show that a closed-form solution is also achievable using the minimax formulation in (2). Again we are only interested in the nontrivial case where $\min \left\{P_{i}\right\}<$ $P_{N+1}<\sum_{i=1}^{N} P_{i}$.

It is easy to see that for MISO channels, $\overline{\mathbf{S}}$ in (3) becomes a scalar and thus maximization of $f(\mathbf{q}, \overline{\mathbf{S}})$ with $\overline{\mathbf{S}}$ is always obtained at $\overline{\mathbf{S}}=P=\sum_{i=1}^{N+1} P_{i}$. Thus the minimax problem is reduced to

$$
\begin{aligned}
\min _{\mathbf{q} \geq 0} & \log \frac{\left|\operatorname{diag}\left(q_{N+1}+q\right)+P \mathbf{H}^{\dagger} \mathbf{H}\right|}{\left|\operatorname{diag}\left(q_{N+1}+q\right)\right|} \\
\text { subject to } & \sum_{i=1}^{N+1} P_{i} q_{i}=P
\end{aligned}
$$

which is equivalent to (by noting that $\mathbf{H}$ is a row vector)

$$
\begin{array}{rl}
\min _{\mathbf{q} \geq 0} & \mathbf{H}\left(\operatorname{diag}\left(q_{N+1}+q\right)^{-1} \mathbf{H}^{\dagger}\right. \\
\text { subject to } & \sum_{i=1}^{N+1} P_{i} q_{i}=P .
\end{array}
$$

To make notation clear, let us write $\mathbf{H}=\left[h_{1}, h_{2}, \ldots, h_{N}\right]$. Then (19) is explicitly written as

$$
\begin{aligned}
\min _{\mathbf{q} \geq 0} & \sum_{i=1}^{N} \frac{\left|h_{i}\right|^{2}}{q_{i}+q_{N+1}} \\
\text { subject to } & \sum_{i=1}^{N+1} P_{i} q_{i}=P .
\end{aligned}
$$

Without loss of generality let us assume $\left\{\frac{\left|h_{i}\right|}{\sqrt{P_{i}}}\right\}$ are in decreas-

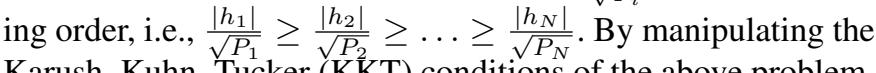
Karush-Kuhn-Tucker (KKT) conditions of the above problem, we arrive at the following closed-form solution

$$
\begin{gathered}
q_{N+1}=\frac{1}{\sqrt{\gamma}} \sqrt{\frac{\sum_{i=k+1}^{N}\left|h_{i}\right|^{2}}{\left(P_{T}-\sum_{i=1}^{k} P_{i}\right)}} \\
q_{i}=\frac{1}{\sqrt{\gamma}}\left(\frac{\left|h_{i}\right|}{\sqrt{P_{i}}}-\sqrt{\frac{\sum_{i=k+1}^{N}\left|h_{i}\right|^{2}}{\left(P_{T}-\sum_{i=1}^{k} P_{i}\right)}}\right), i=1, \ldots, k \\
q_{i}=0, i=k+1, k+2, \ldots, N
\end{gathered}
$$

where $k$ is the least solution to the following inequality:

$$
\frac{\sum_{i=k+1}^{N}\left|h_{i}\right|^{2}}{P_{T}-\sum_{i=1}^{k} P_{i}} \geq \frac{\left|h_{N}\right|^{2}}{P_{N}}
$$

and

$\gamma=\left(\frac{\sum_{i=1}^{k}\left|h_{i}\right| \sqrt{P_{i}}+\left(P_{T}-\sum_{i=1}^{k} P_{i}\right) \sqrt{\frac{\sum_{i=k+1}^{N}\left|h_{i}\right|^{2}}{\left(P_{T}-\sum_{i=1}^{k} P_{i}\right)}}}{P}\right)^{2}$.

The detailed derivation of this closed-form solution is omitted due to space limitations. 


\section{NUMERICAL RESULTS}

In this section, we evaluate the performance of the proposed algorithm by numerical experiments. More specifically, we focus on the important case of joint SPC and PAPC. We consider here the most common case encountered in practice, where each transmit antenna is subject to the same power constraint, i.e., $P_{0}$, for $i=1,2, \ldots, N$. Further, as mentioned earlier, we are only interested in the case where $P_{0}<P_{T}<N P_{0}=P_{A}$. Unless stated otherwise, the error tolerances $\epsilon_{1}$ and $\epsilon_{2}$ are set to $10^{-6}$ for all simulations. Other relevant simulation parameters are specified for each setup. Note that any average result is obtained over 1000 i.i.d. channel realizations. The MATLAB code was executed on a 64-bit desktop that supports 8 Gbyte RAM and Intel CORE i7.

First, we study the convergence properties of the proposed algorithm under SPC, PAPC and interference power constraint. In this experiment, we fix the ratio between the interference power constraint and SPC at 0.5. The convergence rate of Algorithm 2 with different ratios of $P_{T} / P_{A}$ is plotted in the Fig. 1. The residual error is defined as the absolute difference between two consecutive objectives. For the randomly generated channel realization considered in Fig. 1, Algorithm 2 can converge with less than eight iterations and even fewer iterations in high power scenarios. For a fixed $P_{A}$, the convergence results of Algorithm 2 seem to be insensitive to the ratio between $P_{T}$ and $P_{A}$. We also notice that when the solution is close to the optimal one, the proposed algorithm exhibits a superlinear convergence.

Next, we report the average run time of Algorithm 2 along with available free and commercial interior-point solvers e.g., SEDUMI [21] and MOSEK [22]. Both the solvers are executed through the parser YALMIP [23]. The ratio $P_{T} / P_{A}$ is set to 0.8 . The symbol $\times$ denotes a case where the run time is extremely high or where the solvers could not run successfully due to insufficient memory. As can be seen clearly from Table I, interior-point method based convex solvers are not suitable for large-scale MIMO systems because their complexity can increase rapidly with the problem size which results in prohibitive overall computation time. Meanwhile, the proposed algorithm shows a consistently low run time.

Finally, we compare the average capacity of MIMO systems under different power constraint settings i.e., SPC, PAPC, and joint SPC and PAPC. As shown in Fig. 2, the capacity under joint SPC and PAPC is lower than that of PAPC because in this case, the maximum is achieved at a point where not all PAPCs are satisfied with equality. We also observe that when the PAPC is set to be equal for all transmit antennas, the capacity of PAPC is close to the one under SPC as previously observed in [6], [14].

\section{CONCLUSIONS}

We have proposed efficient algorithms for computing the MIMO capacity under multiple LTCCs. We have first transformed the maximization problem in the $\mathrm{BC}$ into an equivalent minimax problem in the dual MAC. Then, CCP together with AO have been utilized to derive a closed-form solution. Our

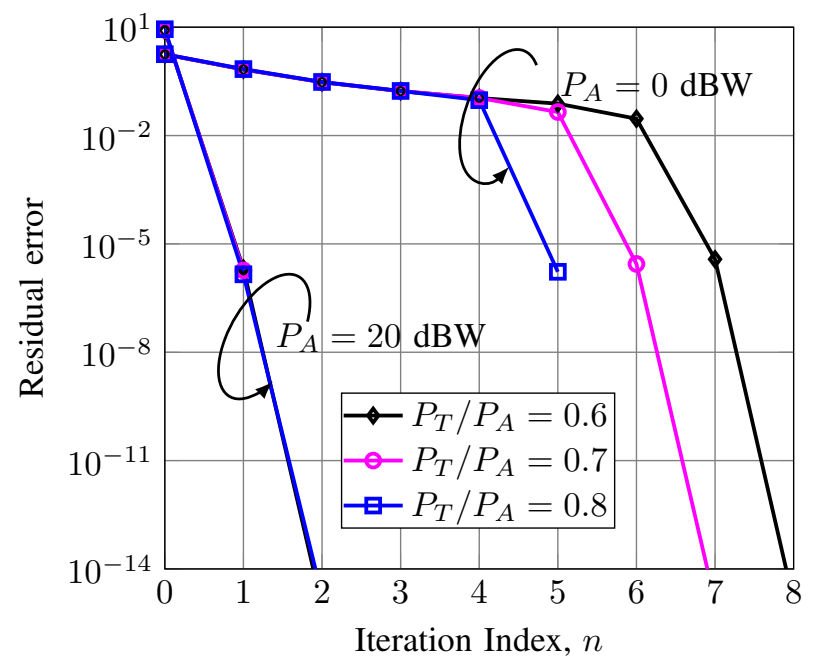

Fig. 1. Convergence rate of the proposed algorithm for SU-MIMO under multiple power constraints i.e., SPC, PAPC and interference power constraint with the number of transmit antennas $N=2$ and the number of receive antennas $M=8$. The interference power constraint is set to half of the SPC.

TABLE I

Average Run time (SECONDS) COMPARison with $P_{T} / P_{A}=0.8$, NUMBER OF RECEIVE ANTENNAS $M=2$. THE RUN TIME IS AVERAGED OVER 1000 CHANNEL REALIZATIONS.

\begin{tabular}{c|c|c|c|c|c|c}
\hline \multirow{2}{*}{$P_{A}$} & \multirow{2}{*}{ Algorithms/solvers } & \multicolumn{5}{|c}{ No. of transmit antennas $N$} \\
\cline { 3 - 7 } & & 8 & 16 & 32 & 64 & 128 \\
\hline \multirow{3}{*}{ 0dBW } & Algorithm 2 & $\mathbf{0 . 0 4 8}$ & $\mathbf{0 . 0 8 9}$ & $\mathbf{0 . 2 4 3}$ & $\mathbf{0 . 6 7 6}$ & $\mathbf{2 . 3 7 1}$ \\
\cline { 2 - 7 } & SEDUMI & 0.058 & 0.225 & 3.485 & $\times$ & $\times$ \\
\cline { 2 - 7 } & MOSEK & 0.007 & 0.040 & 0.588 & $\times$ & $\times$ \\
\hline \hline \multirow{3}{*}{ 10dBW } & Algorithm 2 & $\mathbf{0 . 0 3 7}$ & $\mathbf{0 . 0 8 2}$ & $\mathbf{0 . 2 4 2}$ & $\mathbf{0 . 6 9 0}$ & $\mathbf{2 . 2 6 2}$ \\
\cline { 2 - 7 } & SEDUMI & 0.057 & 0.247 & 3.957 & $\times$ & $\times$ \\
\cline { 2 - 7 } & MOSEK & 0.007 & 0.047 & 0.612 & $\times$ & $\times$ \\
\hline
\end{tabular}

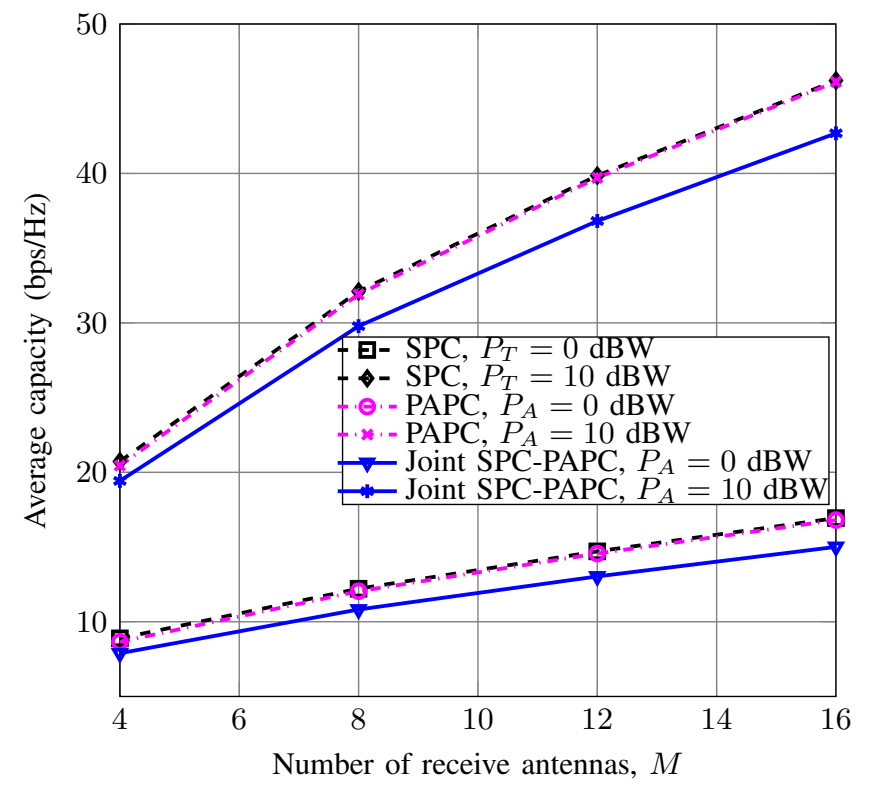

Fig. 2. Average capacity with the number of transmit antennas $N=16$, $P_{T} / P_{A}=0.8$ for joint SPC and PAPC. 
analysis and numerical results have demonstrated that this proposed approach has low complexity, is highly scalable, and thus is suitable to study the capacity of very large-scale MIMO systems.

\section{APPENDIX PROOF OF THEOREM 1}

In this appendix, we prove the duality transformation in (3). We first write the partial Lagrangian function of (2) as

$$
\begin{aligned}
\mathcal{L}(\mathbf{a}, \mathbf{S}) & =\log \left|\mathbf{I}+\mathbf{H S H}^{\dagger}\right|-\sum_{i} a_{i}\left(\operatorname{tr}\left(\mathbf{E}_{i} \mathbf{S}\right)-P_{i}\right) \\
& =\log \left|\mathbf{I}+\mathbf{H} \mathbf{S} \mathbf{H}^{\dagger}\right|-\operatorname{tr}(\mathbf{B S})+\mathbf{p}^{T} \mathbf{a}
\end{aligned}
$$

where $\mathbf{B}=\sum_{i} a_{i} \mathbf{E}_{i}, \mathbf{a}=\left[a_{1}, a_{2}, \ldots, a_{i}, \ldots, a_{L}\right]^{T}$. Note that $\mathbf{B}$ must be positive definite (and therefore invertible), otherwise $\max _{\mathbf{S} \succ 0} \mathcal{L}(\mathbf{a}, \mathbf{S}) \rightarrow \infty$. Let $\hat{\mathbf{S}}=\mathbf{B}^{1 / 2} \mathbf{S B}^{1 / 2}$, then (26) becomes

$$
\mathcal{L}(\mathbf{a}, \mathbf{S})=\log \left|\mathbf{I}+\mathbf{H B}^{-1 / 2} \hat{\mathbf{S B}} \mathbf{B}^{-1 / 2} \mathbf{H}^{\dagger}\right|-\operatorname{tr}(\hat{\mathbf{S}})+\mathbf{p}^{T} \mathbf{a} .
$$

Denote $\mathbf{U} \boldsymbol{\Sigma} \mathbf{V}^{\dagger}$ be the singular value decomposition of $\mathbf{H B}^{-1 / 2}$, i.e., $\mathbf{U} \boldsymbol{\Sigma} \mathbf{V}^{\dagger}=\mathbf{H} \mathbf{B}^{-1 / 2}$, we proceed with the introduction of dual objective:

$$
\mathcal{D}(\mathbf{a})=\max _{\dot{\mathbf{S}} \succeq 0} \log \left|\mathbf{I}+\mathbf{B}^{-1 / 2} \mathbf{H}^{\dagger} \dot{\mathbf{S}} \mathbf{H} \mathbf{B}^{-1 / 2}\right|-\operatorname{tr}(\dot{\mathbf{S}})+\mathbf{p}^{T} \mathbf{a}
$$

where the relationship between $\hat{\mathbf{S}}$ and $\dot{\mathbf{S}}$ is given by $\hat{\mathbf{S}}=$ $\mathbf{V U}^{\dagger} \dot{\mathbf{S}} \mathbf{U} \mathbf{V}^{\dagger}$.

By definition, the dual problem is $\min _{\mathbf{a} \geq 0} \mathcal{D}(\mathbf{a})$, or equivalently

$$
\min _{\mathbf{a} \geq 0} \max _{\dot{\mathbf{S}} \succeq 0} \log \frac{\left|\mathbf{B}+\mathbf{H}^{\dagger} \dot{\mathbf{S}} \mathbf{H}\right|}{|\mathbf{B}|}-\operatorname{tr}(\dot{\mathbf{S}})+\mathbf{p}^{T} \mathbf{a} .
$$

We can introduce a new optimization $\delta>0$ so that the problem above can be rewritten as

$$
\begin{aligned}
\min _{\mathbf{a} \geq 0, \delta>0} \max _{\dot{\mathbf{S}} \succeq \mathbf{0}} & \log \frac{\left|\mathbf{B}+\mathbf{H}^{\dagger} \dot{\mathbf{S}} \mathbf{H}\right|}{|\mathbf{B}|}-\delta P+\mathbf{p}^{T} \mathbf{a} \\
\text { subject to } & \operatorname{tr}(\dot{\mathbf{S}}) \leq \delta P .
\end{aligned}
$$

Again, we can change the variables by

$$
\hat{q}_{i}=\frac{a_{i}}{\delta}, \overline{\mathbf{S}}=\frac{\dot{\mathbf{S}}}{\delta},
$$

Substituting (31) into (30), we arrive at the following optimization problem

$$
\begin{aligned}
\min _{\hat{\mathbf{q}} \geq \mathbf{0}} \max _{\overline{\mathbf{S}} \succeq \mathbf{0}} & \log \frac{\left|\sum_{i} \hat{q}_{i} \mathbf{E}_{i}+\mathbf{H}^{\dagger} \overline{\mathbf{S}} \mathbf{H}\right|}{\left|\sum_{i} \hat{q}_{i} \mathbf{E}_{i}\right|} \\
\text { subject to } & \operatorname{tr}(\overline{\mathbf{S}}) \leq P \\
& \mathbf{p}^{T} \hat{\mathbf{q}} \leq P
\end{aligned}
$$

\section{REFERENCES}

[1] E. Telatar, "Capacity of multi-antenna Gaussian channels," Eur. Trans. Telecommun, vol. 10, pp. 585-598, Nov. 1999.

[2] G. J. Foschini and M. J. Gans, "On limits of wireless communications in a fading environment when using multiple antennas," Wireless Pers.Commun, vol. 6, pp. 311-335, Mar. 1998.

[3] W. Yu, W. Rhee, S. Boyd, and J. Cioffi, "Iterative water-filling for Gaussian vector multiple-access channels," IEEE Trans. Inf. Theory, vol. 50, no. 1, pp. 145-152, Jan. 2004.

[4] N. Jindal, W. Rhee, S. Vishwanath, S. A. Jafar, and A. Goldsmith, "Sum power iterative water-filling for multi-antenna Gaussian broadcast channels," IEEE Trans. Inf. Theory, vol. 51, no. 4, pp. 1570-1580, Apr. 2005.

[5] M. Vu, "MISO capacity with per-antenna power constraint," IEEE Trans. Commun., vol. 59, no. 5, pp. 1268-1274, May 2011.

[6] _ "MIMO capacity with per-antenna power constraint," in Proc. IEEE GLOBECOM, Dec. 2011, pp. 1 - 5 .

[7] W. Yu and T. Lan, "Transmitter optimization for the multi-antenna downlink with per-antenna power constraints," IEEE Trans. Signal Process., vol. 55, no. 6, pp. 2646-2660, Jun. 2007.

[8] T. M. Pham, and R. Farrell, and L.-N. Tran, "Low-complexity approaches for MIMO capacity with per-antenna power constraint," in Proc. IEEE VTC-Spring, Jun. 2017, pp. 1-7.

[9] P. L. Cao, T. J. Oechtering, R. F. Schaefer, and M. Skoglund, "Optimal transmit strategy for MISO channels with joint sum and per-antenna power constraints," IEEE Trans. Signal Process., vol. 64, no. 16, pp. 4296 - 4306, Aug. 2016.

[10] S. Loyka, "The capacity of Gaussian MIMO channels under total and per-antenna power constraints," IEEE Trans. Commun., vol. 65, no. 3 , pp. 1035 - 1043, Mar. 2017.

[11] L. Zhang, R. Zhang, Y. C. Liang, Y. Xin, and H. V. Poor, "On Gaussian MIMO BC-MAC duality with multiple transmit covariance constraints," IEEE Trans. Inf. Theory, vol. 58, no. 4, pp. 2064 - 2078, Apr. 2012.

[12] H. Huh, H. C. Papadopoulos, and G. Caire, "Multiuser MISO transmitter optimization for intercell interference mitigation," IEEE Trans. Signal Process., vol. 58, no. 8, pp. 4272 - 4285, Aug. 2010.

[13] Y. Yang, G. Scutari, P. Song, and D. P. Palomar, "Robust MIMO cognitive radio systems under interference temperature constraints," IEEE J. Sel. Areas Commun., vol. 31, no. 11, pp. 2465-2482, Nov. 2013.

[14] T. M. Pham, and R. Farrell, and L.-N. Tran, "Alternating optimization for capacity region of Gaussian MIMO broadcast channels with perantenna power constraint," in Proc. IEEE VTC-Spring, Jun. 2017, pp. $1-6$.

[15] W. Yu, "Uplink-downlink duality via minimax duality," IEEE Trans. Inf. Theory, vol. 52, no. 2, pp. 361-374, Feb. 2006.

[16] A. Beck, A. Ben-Tal, and L. Tetruashvili, "A sequential parametric convex approximation method with applications to nonconvex truss topology design problems," Journal of Global Optimization, vol. 47, no. 1 , pp. 29-51, 2010

[17] L. Condat, "Fast projection onto the simplex and the $\ell_{1}$ ball," Mathematical Programming, Series A, vol. 158, no. 1, pp. 575 - 585, Jul. 2016.

[18] L. Armijo, "Minimization of functions having Lipschitz continuous first partial derivatives." Pacific J. Math., vol. 16, no. 1, pp. 1 - 3, 1966.

[19] R. L. Burden and J. D. Faires, Numerical analysis, 9th ed. Brooks Cole, 2011.

[20] G. H. Golub and C. F. V. Loan, Matrix Computations, 4th ed. The John Hopkins Univ. Press, 2013.

[21] J. F. Sturm, "Using SeDuMi 1.02, a MATLAB toolbox for optimization over symmetric cones," Optimization Methods and Software, vol. 11-12, pp. 625-653, 1999.

[22] M. ApS, The MOSEK optimization toolbox for MATLAB manual. Version 7.1 (Revision 28)., 2015.

[23] J. Löfberg, "YALMIP : A toolbox for modeling and optimization in MATLAB," in Proc. the CACSD Conference, 2004.

which completes the proof. 